\title{
Gesamterneuerungswahlen 2016: Porträts der Kandidierenden
}

Im Rahmen der Ärztekammer vom 28. April 2016 finden die Gesamterneuerungswahlen aller Organe der FMH für eine neue Legislatur statt. 15 der bei Redaktionsschluss bekannten Kandidatinnen und Kandidaten für den Zentralvorstand, die Geschäftsprüfungskommission sowie das Präsidium und Vizepräsidium der Standeskommission stellen sich im Folgenden in einem Porträt vor.

\section{Wen wählt die Ärztekammer?}

Gewählt werden an der Ärztekammer vom 28. April 2016 die sieben Mitglieder des Zentralvorstandes der FMH inklusive Präsident und zwei Vizepräsidenten, der Präsident des SIWF, die fünf Mitglieder der Geschäftsprüfungskommission, der Präsident und die Vizepräsidenten der Standeskommission sowie die Mitglieder der Delegiertenversammlung. Grundvoraussetzung für die Wählbarkeit einer Kandidatin, eines Kandidaten ist u.a. der Arztberuf sowie die FMH-Mitgliedschaft. Bei den Kandidaten für den Zentralvorstand wird von der Ärztekammer zusätzlich die Erfüllung eines Anforderungsprofils gefordert.

\section{Christoph Bosshard (bisher)}

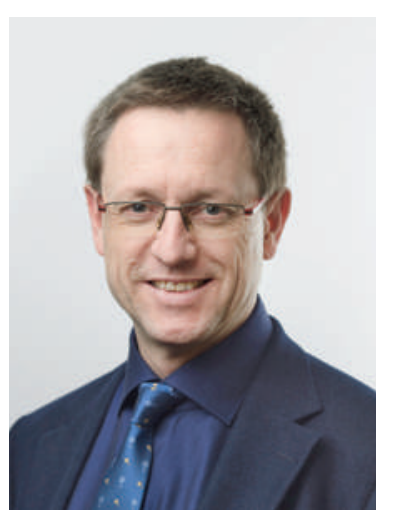

Titel: Dr. med.

Geburtsjahr: 1964

Wohnort: Bern

Bürgerort: Turbenthal

Zivilstand: verheiratet

Facharzttitel: Orthopädische Chirurgie und

Traumatologie des Bewegungsapparates $(\mathrm{CH})$,

Zertifizierter Medizinischer Gutachter SIM
E-Mail: christoph.bosshard[at]hin.ch

sion Versicherungsmedizin), Berner Gesellschaft der Orthopädischen Chirurgen (Vizepräsident)

\section{Interessenbindungen}

Versicherungsmedizin Suva

Weitere Qualifikationen

Lehrauftrag für Versicherungsmedizin Universität Bern

\section{Sprachen}

Deutsch, Französisch und Englisch fliessend; Italienisch mündlich

\section{Aktuelle berufliche Tätigkeit}

Mitglied bei medizinischen Gesellschaften Verband Schweizerischer Assistenz- und Oberärztinnen und -ärzte VSAO (Geschäftsausschuss), swiss orthopaedics (Mitglied Standeskommission / Sub-Kommis-
Leiter Versicherungsmedizin Suva Bern/Fribourg/Sion/ Solothurn, Vizepräsident der FMH, Departementsverantwortlicher Daten, Demographie und Qualität der FMH 
Parteizugehörigkeit

Keine

Bisherige politische Mandate

Keine

Bisherige standespolitische Mandate

Präsident Verband Schweizerischer Assistenz- und Oberärztinnen und -ärzte VSAO, Präsident VSAO Sektion Bern

\section{Führungserfahrung}

Militär: Kompanie-Kommandant, Bataillons-Arzt; Medizin: kaderärztliche Tätigkeit Orthopädische Kliniken Spital Netz Bern inklusive Sonderaufgaben; Leiter Versicherungsmedizin Suva-Agenturen Bern/Fribourg/ Sion/Solothurn mit insgesamt 28 Mitarbeitenden über die erwähnten vier Standorte verteilt; Führungsausbildungscurriculum der Suva

\section{Motivation}

Nach meiner ersten Legislatur als Departementsverantwortlicher Daten/Demographie/Qualität und seit Oktober 2015 als Vizepräsident der FMH blicke ich auf eine reichhaltige Zeit gefüllt mit Herausforderungen, Erfahrungen und (Teil-)Erfolgen zurück. Durch die Bündelung der Qualitätsaktivitäten in der SAQM wurde nebst dem Synergie-Nutzen auch ein deutlicher Mehrwert im Bereich Wissenstransfer erzielt. Mit un serem Pilot-Projekt «Sektorenübergreifender Behandlungspfad Kolonkarzinom» erreichten wir die Koordination von zehn ärztlichen und elf nicht-ärztlichen Fachgesellschaften und ernteten internationale Anerkennung. Die Themen Daten und Demographie wurden im Büro Daten/Demographie gebündelt und damit mehrere Arbeitsgruppen aufgehoben. Dank der Mithilfe ihrer Mitglieder verfügt die FMH in Form der myFMH-Daten über eine Grundlage, welche durch keine andere zu überbieten ist. Gerne stelle ich mich zur Verfügung, um die begonnenen Arbeiten weiterzuführen - gemeinsam mit Ihnen, sehr geehrte Kolleginnen und Kollegen! 


\section{Monique Gauthey (bisher)}

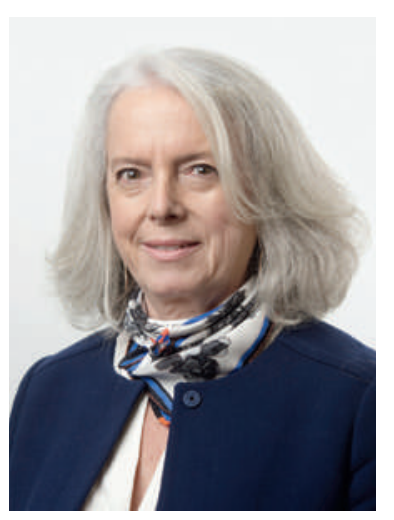

Geburtsjahr: 1953

Wohnort: Cologny (Genf)

Bürgerort: Arnex s/Orbe

Zivilstand: verheiratet, Mutter von drei Kindern

E-Mail: monique.gauthey[at]bluewin.ch und

monique.gauthey[at]hin.ch

Facharzttitel: Kinder- und Jugendpsychiatrie und

-psychotherapie
Mitgliedschaft bei medizinischen Gesellschaften Schweizerische Gesellschaft für Kinder- und Jugendpsychiatrie und -Psychotherapie SGKJPP, Schweizerische Gesellschaft für Forensische Psychiatrie, Schweizerische Gesellschaft für Psychotraumatologie (SSPT), Medical Women Switzerland MWS.

\section{Interessenbindungen \\ Keine}

\section{Sprachen}

Muttersprache Französisch, sehr gute Deutsch- und Englischkenntnisse, Italienischkenntnisse

\section{Aktuelle berufliche Tätigkeit}

Tätigkeit in Privatpraxis (seit 2000), Referenzärztin des Sekretariats für Sonderpädagogik der Erziehungsdirektion (SPS - DIP) des Kantons Genf

\section{Parteizugehörigkeit}

Keine

\section{Bisherige standespolitische Mandate}

Mitglied des Zentralvorstands der FMH seit Juni 2008 (Ressort eHealth, anschliessend Spitalärztinnen und -ärzte), Ärztekammer seit 2005 und Delegiertenversammlung seit 2007, Vorstand der SGKJPP seit 2005, später Co-Präsidentin der SGKJPP (von 2006 bis 2008)

\section{Führungserfahrung}

Oberärztin mit Verantwortung für die Liaisonpsychiatrie und die stationäre Kinderpsychiatrie im Kinderspital des HUG (von 1990 bis 1999)

\section{Motivation}

Nach zwei Amtszeiten im Zentralvorstand scheint mir die aktive Einbeziehung der Ärztinnen und Ärzte in die Gesundheitspolitik wichtiger denn je. Angesichts des Regulierungsdrucks von Seiten der Politik muss die Ärzteschaft ihren Standpunkt in vielen Bereichen mit Nachdruck vertreten. Der europäische Vergleich zeigt, dass das Risiko einer qualitativen Verschlechterung der medizinischen Leistungen durchaus real ist und die Ärzteschaft unbedingt wachsam bleiben muss. Ob im Hinblick auf das Tarifsystem für medizinische Leistungen, den Einsatz neuer Informationstechnologien und die Gefahren angesichts von Big Data - die Themen sind vielfältig. Die Auswirkung der Sicherheitsmassnahmen auf die Vertraulichkeit, die Einmischung in das Arzt-Patienten-Verhältnis durch Dritte wie Versicherer oder die Folgen der Migration für das Gesundheitssystem - die nächste Legislaturperiode wird grosse Herausforderungen für uns mit sich bringen. Auch die Vertretung von Minderheiten im Zentralvorstand liegt mir am Herzen. 


\section{Yvonne Gilli}

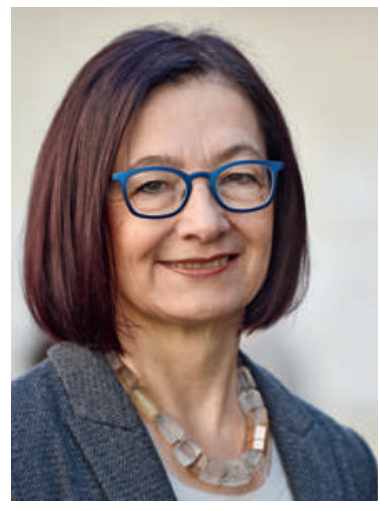

Titel: Dr. med.

Geburtsjahr: 1957

Wohnort: Wil SG

Bürgerort: Neudorf LU

Zivilstand: verheiratet, drei Söhne

E-Mail: yvonne.gilli[at]hin.ch

Facharzttitel: Allgemeine Innere Medizin

\section{Fachmitgliedschaften}

Schweizerische Gesellschaft für Allgemeine Innere Medizin (SGAIM); Schweizerische Gesellschaft für Ultraschall in der Medizin (SGUM); Assoziation Schweizer Ärztegesellschaften für Akupunktur und Chinesische Medizin (ASA); Schweizerischer Verein homöopathischer Ärztinnen und Ärzte (SVHA); Ärztinnen und Ärzte für Umweltschutz (AefU)

Interessenbindungen

Verwaltungsrätin xundart AG, Beirätin reliva AG

\section{Weitere Qualifikationen}

Handelsdiplom, dipl. Pflegefachfrau AKP

\section{Sprachen}

Muttersprache Deutsch, Englisch sehr gut, Französisch, Italienisch

\section{Aktuelle berufliche Tätigkeit}

Führung einer Gruppenpraxis mit den Schwerpunkten Komplementärmedizin, Gynäkologie und psychologische Beratung; Verwaltungsrätin Ärztenetzwerk xundart AG

\section{Parteizugehörigkeit \\ Grüne Partei Schweiz}

\section{Politische Mandate}

2007-2015: Nationalrätin und Mitglied folgender nationalrätlicher Kommissionen: soziale Sicherheit und Gesundheit; Wissenschaft, Bildung und Kultur; Finanzen; 2004-2007: Kantonsrätin SG; 2000-2005: Mitglied Stadtparlament Wil SG
Bisherige standespolitische Mandate

Keine

\section{Führungserfahrung}

Mehrjährige Führung einer Gruppenpraxis in Wil; Gründung, Aufbau und Führung (Geschäftsleitung und Mitglied Verwaltungsrat) des Ärztenetzwerkes xundart AG (gemischtes Netzwerk mit Spezialisten und Hausärzten)

Führungserfahrung NGO-Ebene: Stiftungsratspräsidentin «Sexuelle Gesundheit Schweiz» (Dachorganisation der kantonalen und kommunalen Familienberatungsstellen); Grüne Partei: kantonales Präsidium, Vize-Präsidium Bundeshausfraktion; Präsidium «Patientenstelle Ostschweiz»

\section{Motivation}

Während meiner nationalrätlichen Tätigkeit war es mir wichtig, mich standespolitisch für die FMH zu engagieren. Der Arztberuf ist eine Herausforderung, wegen des ökonomischen Drucks und wegen zunehmender Interessenkonflikte. Pflegefachleute und Psychologen sind unterwegs zu eigenständigen Leistungserbringern. Die Apotheker verlangen eine Beteiligung an der medizinischen Grundversorgung. Gleichzeitig wird das Gesundheitswesen zunehmend komplexer. Nur eine wirksame und professionelle Standespolitik verhindert, dass die Ärzteschaft wegen konkurrierender Interessen politischen Entscheidungsträgern als Sündenbock dient.

Erfolgreiche Standespolitik setzt eine gute Vernetzung voraus, welche über die eigenen Partei- und Berufsgrenzen reicht. Meine berufliche Laufbahn mit dem Medizinstudium auf dem zweiten Bildungsweg und meine langjährige politische Erfahrung auf kommunaler, kantonaler und nationaler Ebene zeichnen meine Fähigkeiten in diesen Bereichen aus. Ich würde meine Erfahrung gern uneingeschränkt der FMH zur Verfügung stellen. 


\section{Jean-Baptiste Ollyo}

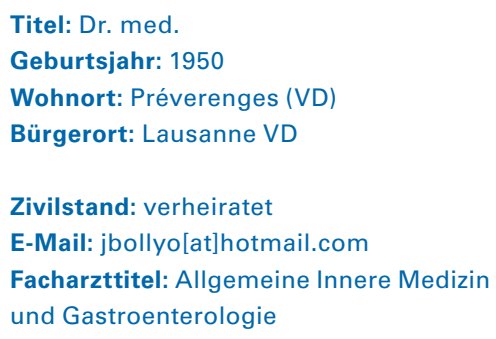

\section{Mitglied bei medizinischen Gesellschaften}

Ärztegesellschaft des Kantons Waadt, Schweizerische Gesellschaft für Gastroenterologie, Schweizerische Gesellschaft für Ultraschall in der Medizin, Fraternité AghPan (humanistische Medizin unter Wahrung der staatsbürgerlichen Verantwortung)

\section{Interessenbindungen}

Keine Angabe

Weitere Qualifikationen

Medizinrecht

Sprachen

Französisch, Italienisch, Englisch, gute Deutschkenntnisse

\section{Aktuelle berufliche Tätigkeit}

Arzt in Teilzeit in einem medizinischen Zentrum, Verfassen eines Buches über konstitutionelle Magerkeit

\section{Parteizugehörigkeit}

FDP. Die Liberalen

Bisherige politische Mandate

Keine Angabe
Bisherige standespolitische Mandate

Keine Angabe

\section{Führungserfahrung}

Keine Angabe

\section{Motivation}

Ich kandidiere für den Zentralvorstand der FMH, um:

1. die Macht wieder an alle Mitglieder zurückzugeben (obligatorische Urabstimmung, wenn ein Beschluss zu ethischen Aspekten ansteht, der für die Mitglieder verbindlich ist);

2. die legitimen beruflichen und wirtschaftlichen Interessen aller Mitglieder zu vertreten und dafür zu sorgen, dass sich die FMH tatsächlich für die Ehre, Freiheit und Unabhängigkeit des Arztberufs einsetzt;

3. die strikte Gewaltentrennung wiederherzustellen (beispielsweise Aufhebung der legislativen und richterlichen Befugnisse des FMH-Präsidenten);

4. die moralische Autorität der FMH zu stärken, damit sie für alle wirklich glaubwürdig ist, einschliesslich für unsere externen Partner;

5. die Information zu entwickeln und zu verbessern;

6. die Befugnisse der Standeskommission der FMH zu erweitern, damit sie bei Bedarf in der Lage ist, über die kantonalen Ärztegesellschaften und die Organe der FMH zu «urteilen».

7. auch die Nichtmitglieder der FMH zu schützen (die nicht konsultiert werden, aber trotzdem verschiedenen Beschlüssen unterworfen sind). 


\section{Remo Osterwalder (bisher)}

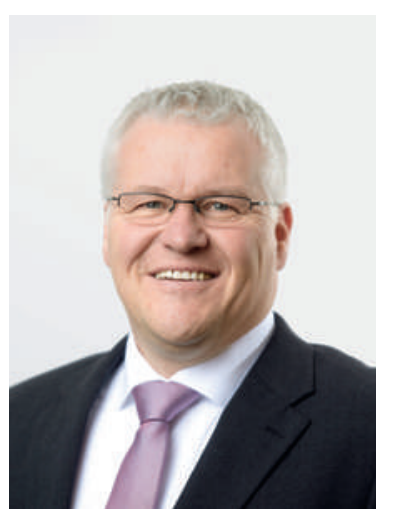

Titel: Dr. med.

Geburtsjahr: 1966

Wohnort: 2800 Delémont

Bürgerort: Gaiserwald SG, Basel

Zivilstand: verheiratet, zwei Kinder

E-Mail: osterwalderr@bluewin.ch

Facharzttitel: Kardiologie
Mitglied bei medizinischen Gesellschaften

Schweizerische Gesellschaft für Kardiologie, Société Médicale du Canton du Jura (SMCJU), European Society of Cardiology (ESC)

\section{Interessenbindungen}

Verwaltungsratspräsident der Juramed AG und der CDS Centre de Santé Delémont AG, Mitglied der Eidgenössischen Kommission für Analysen, Mittel und Gegenstände (EAMGK), Mitglied des Verwaltungsrats von FMH Services (Genossenschaft und Consulting AG)

\section{Weitere Qualifikationen}

Wirtschaftliche Ausbildung in Administration, Gründer eines Ärztezentrums

\section{Sprachen}

Französisch, Deutsch, Englisch

Aktuelle berufliche Tätigkeit

Praxisinhaber und Konsiliararzt am Kantonsspital Baselland Laufen und Hôpital du Jura

\section{Bisherige politische Mandate \\ Keine}

Bisherige standespolitische Mandate

Mitglied des Zentralvorstands der FMH seit 2010, Präsident der Société Médicale du Canton du Jura (SMCJU) seit 2008

\section{Führungserfahrung}

Mitglied des Zentralvorstands der FMH, Projektleiter der CDS Centre de Santé Delémont AG, Chef einer Gruppenpraxis, Ausbildungsverantwortlicher für Assistenzärztinnen und -ärzte

\section{Motivation}

Seit sechs Jahren im Zentralvorstand der FMH, setzte ich mich für die Interessen und Anliegen der freipraktizierenden Ärzteschaft ein. Nicht nur theoretisch, sondern auch praktisch zeige ich als Projektleiter Lösungsansätze für die zukünftige medizinische Versorgung vor allem in peripheren Regionen auf. Im Vordergrund stehen zeitgemässe Arbeitsmodelle für die aktuellen und nachfolgenden Generationen. Projekte in diesem Zusammenhang sind für die Ärzteschaft wichtig, insbesondere bei der Nachfolgeregelung und für den Erhalt der freien Berufsgattung. Eine neue Herausforderung ist die aktive Mitarbeit als Mitglied der Eidgenössischen Kommission für Analysen, Mittel und Gegenstände (EAMGK). Mit meiner Wahl in den Verwaltungsrat der Genossenschaft FMH Services und FMH Consulting Services AG hat eine erneute Annäherung zwischen dem Zentralvorstand und der ärzteeigenen Institution stattgefunden. Ich bin bereit für ein weiteres Mandat und darf dabei auf das über die Jahre stetig gewachsene politische und berufliche Netzwerk zurückgreifen.

\section{Parteizugehörigkeit}

Parteilos 


\section{Gert Printzen (bisher)}

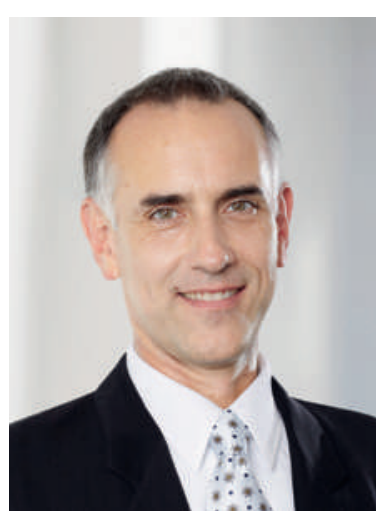

Titel: Dr. med. et Dipl. Biochem.

Geburtsjahr: 1956

Wohnort: Luzern

Bürgerort: Luzern

Zivilstand: ledig

E-Mail: g.printzen[at]analytica.ch

Facharzttitel: Labormedizin; FAMH mult. (Immuno-

logie, Klinische Chemie, Mikrobiologie)

\section{Mitglied bei medizinischen Gesellschaften}

Verband Schweizer. Assistenz- und Oberärztinnen und -ärzte (VSAO); Verband der medizinischen Laboratorien der Schweiz (FAMH); Schweizer. Gesellschaften für Medizinische Mikrobiologie (SGM), Klinische Chemie (SGKC) und Klinische Immunologie und Allergologie (SGAI); American Association for Clinical Chemistry (AACC); International Society for Infectious Diseases (ISID)

Interessenbindungen

Keine

\section{Weitere Qualifikationen}

Interner Auditor; Business Excellence Assessor mit EFQM-Zertifikat; diverse Kaderführungskurse

\section{Sprachen}

Deutsch (Muttersprache), Englisch (fliessend in Wort und Schrift), Französisch (gute Kenntnisse in Wort und Schrift), Spanisch (Grundkenntnisse in Wort und Schrift)

\section{Aktuelle berufliche Tätigkeit}

Abteilungsleiter Medizinische Mikrobiologie und CoLeitung Casemanagement der Analytica Medizinische Laboratorien AG

\section{Parteizugehörigkeit}

Keine

\section{Bisherige politische Mandate}

Keine
Bisherige standespolitische Mandate

Seit 1992: im Vorstand Sektion VSAO Zentralschweiz (u.a. sechs Jahre als Präsident); seit 1994: Zentralvorstand VSAO; seit 1998: Geschäftsausschussmitglied des Zentralverbandes VSAO; seit 2003: Stiftungsrat der Pensionskasse I des VSAO; Mai 2010: Wahl in den Zentralvorstand der FMH, seither Departementsverantwortlicher Heilmittel, Co-Leitung des Departements eHealth, seit März 2015 zusätzlich Departementsverantwortung Paramedizinische Berufe; seit 2010: Vizepräsident der Prüfungskommission Labormedizin FAMH; seit 2012: Stiftungsrat des Toxzentrums Zürich; seit 2013: Mitglied der Standeskommission des VSAO und des SAMW Senats; November 2014: Wahl zum Mitglied des Zürcher Schiedsgerichts in Sozialversicherungsstreitigkeiten

\section{Führungserfahrung}

Über zehn Jahre Abteilungsleitung des Instituts für Klinische Chemie am Inselspital mit bis zu 42 Mitarbeitenden; vierjährige Führungs- und Budget-Verantwortung als Chef des Institut für Klinische Chemie und Immunologie des Luzerner Kantonsspitals; zudem vielfältige Führungserfahrungen in kleineren Teams als Abteilungsleiter und Supervisor der Analytica AG

\section{Motivation}

Flexibel reagieren auf nicht nur gesundheitspolitisch sich verändernde Bedingungen sowie zuverlässig und eigeninitiativ handeln sind Eigenschaften, die «meinen» Departementen in der vergangenen Legislatur zugutekamen. Dazu gehör(t)en u.a. berufsgruppenübergreifende Kooperationen. Bei der Entwicklung der Nationalen Strategien (u.a. NOSO) wurde die FMH somit gut positioniert. Während deren anstehenden Umsetzung will ich neben den gesetzlichen Vorgaben auch ökonomische Erfordernisse einbeziehen und auf Praxistauglichkeit achten. Dies gilt auch für die Ausgestaltung des elektronischen Patientendossiers. Hauptziele im Departement Paramedizinische Berufe sind die Revision BiVo/ Bildungsplan MPA und die Umsetzung der MPA-Strategie. Dies alles im Sinne unserer Mitglieder aktiv mitzugestalten und positiv abzuschliessen ist ein Ziel, wie auch weiterhin engagiert mein Wissen und meine fast 25-jährige standespolitische Erfahrung zum Nutzen der Ärzteschaft in den Zentralvorstand einzubringen sowie Sinnvolles umzusetzen. 


\section{Carlos Beat Quinto}

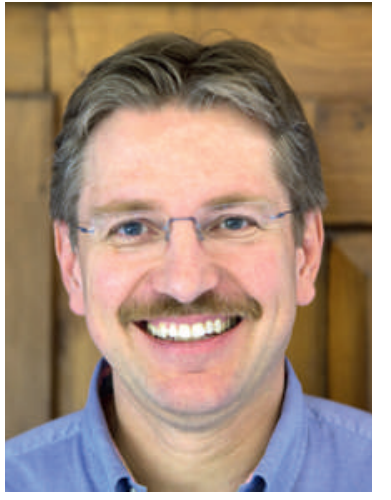

Titel: Dr. med. MPH

Geburtsjahr: 1966

Wohnort: Basel

Bürgerort: Widnau/SG

Zivilstand: verheiratet, ein Sohn

E-Mail: dr.quinto[at]hin.ch; carlos.quinto[at]unibas.ch

Facharzttitel: Allgemeine Innere Medizin

\section{Mitglied bei medizinischen Gesellschaften}

Verband Schweizerischer Assistenz- und Oberärztinnen und -ärzte (VSAO); Schweizerische Gesellschaft für Allgemeine Innere Medizin (SGAIM); Hausärzte Schweiz (MFE); Vereinigung der Hausärztinnen und Hausärzte beider Basel (VHBB); Hausärzteverein Angenstein (HVA); Public Health Schweiz (SSPH - Swiss Society for Public Health); European Public Health Association (EUPHA); Schweizerische Evaluationsgesellschaft (SEVAL); European Evaluation Society (EES)

\section{Interessenbindungen}

Vorstand Ärztegesellschaft BL, Geschäftsleitung Trustcenter syndata

\section{Weitere Qualifikationen}

Master of Public Health; Mitglied der ehemaligen AG Prävention des Kollegiums für Hausarztmedizin KHM (aktuell Fachbereich); Qualitätszirkelmoderator seit 2007

\section{Sprachen}

Deutsch (Muttersprache), Französisch, Englisch, Spanisch, Niederländisch

\section{Aktuelle berufliche Tätigkeit}

hauptberuflich selbständig erwerbend, Partner in der Gemeinschaftspraxis Pfeffingen/BL,zusammen mit Dres. med. J. Fritschi und A. Käppeli; nebenberuflich Lehrbeauftragter am Universitären Zentrum für Hausarztmedizin beider Basel; nebenberuflich «senior scientific collaborator», Schweizerisches Tropen- und
Public Health-Institut (Swiss TPH), Dept. Epidemiology and Public Health, Chronic Disease Epidemiology Unit; Mitglied MINT (Motivational Interviewing Network of Trainers)

\section{Parteizugehörigkeit \\ Parteilos}

\section{Bisherige politischen Mandate Keine}

\section{Bisherige standespolitischen Mandate}

Während der Assistenzzeit mehrjährige Vorstandstätigkeit im Verband Schweizerischer Assistenz- und Oberärztinnen und -ärzte Basel (VBAO) (ehem. VSAO beider Basel): zuerst Vorstandsmitglied, dann Kassier und später Vizepräsident; seit 2012 Mitglied im Ausschuss des Vorstands der Aerztegesellschaft BL; seit 2012 Redaktionsmitglied Synapse (www.emh.ch)

\section{Führungserfahrung}

Personalführung in der Gemeinschaftspraxis; selbständiges Führen von Evaluationsprojekten auf nationaler Ebene; über 10 Jahre Dienst als Stabsarzt (Hauptmann): Organisation des Sanitätsdienstes

\section{Motivation}

Ich möchte die Anliegen der Hausarztmedizin und von Public Health vertreten auf der Basis meiner langjährigen Tätigkeit in der Praxis und an der Universität. In der Hausarztmedizin und im Public-Health-Bereich bin ich interdisziplinäres und interprofessionelles Arbeiten gewohnt, auf kantonaler und nationaler Ebene. Ich verstehe mich als Brückenbauer zwischen Allgemeinmedizin und Public Health, zwischen Medizin aus Individual- und Bevölkerungsperspektive. Mein persönliches Ziel ist es, zur Verbesserung der Kommunikation zwischen den Disziplinen beizutragen, was für alle Ärztinnen und Ärzte von Nutzen sein dürfte. Wichtig ist mir unser ärztlicher Nachwuchs. Deshalb engagiere ich mich in der Aus-, Weiter- und Fortbildung. Die ArztPatienten-Beziehung steht für mich im Zentrum. Der Arztberuf hat auch eine politische Dimension, weshalb ich seit meiner Assistenzzeit standespolitisch tätig bin. Es gilt, die Rahmenbedingungen so mitzugestalten, dass der Arztberuf weiterhin Freude macht. 


\section{Jürg Schlup (bisher)}

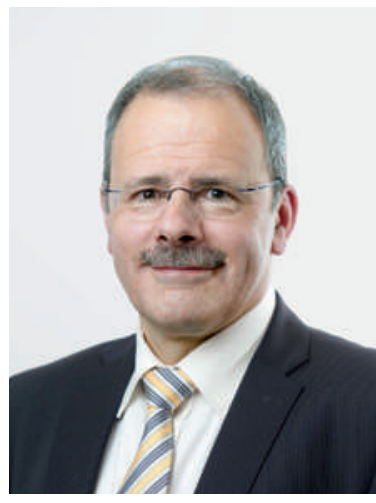

Titel: Dr. med.; Executive MBA HSG

Geburtsjahr: 1955

Wohnort: Zollikofen

Bürgerort: Wengi b. Büren

Zivilstand: verheiratet; zwei Kinder

E-Mail: juerg.schlup[at]fmh.ch

Facharzttitel: Allgemeine Innere Medizin

Mitglied bei medizinischen Gesellschaften

Ärztegesellschaft des Kantons Bern, Schweizerische Gesellschaft für Allgemeine Innere Medizin, Haus- und Kinderärztinnen Schweiz, Verband Schweizerischer Assistenz- und Oberärztinnen und -ärzte

\section{Interessenbindungen}

Mitglied Verwaltungsrat Berner Reha Zentrum AG, Heiligenschwendi, Mitglied Verwaltungsrat Ärztezentrum Oberhasli AG, Meiringen, Patronatskomitee Schweizerische Hirnliga

\section{Weitere Qualifikationen}

Diplom in Unternehmensführung der HSG

\section{Sprachen}

Gemäss Definition CEFR: Deutsch C2; Französisch B2; Englisch B2; Italienisch A2.

\section{Aktuelle berufliche Tätigkeit}

Führung des Berufsverbandes FMH als dessen Präsident

Parteizugehörigkeit

FDP. Die Liberalen

\section{Bisherige politische Mandate}

Mitglied Grosser Gemeinderat Zollikofen 1996-2003, Fraktionsvorsitz, Mitglied Leitender Ausschuss Berner KMU 2006-2011, Vorsitzender Ressort Gesundheit, Vizepräsident Spitalversorgungskommission des Kantons Bern 2008-2010
Bisherige standespolitische Mandate

Präsident Verband Schweizerischer Assistenz- und Oberärztinnen und -ärzte Sektion Bern 1983-1987, Präsident Ärztegesellschaft des Kantons Bern 2001-2010, Mitglied des Stiftungsrats-Ausschusses der Stiftung Patientensicherheit seit 2013, Mitglied des Vorstandes der Schweizerischen Akademie der Medizinischen Wissenschaften seit 2013

\section{Führungserfahrung}

Verbandsführung: zwei kantonale Ärzteverbände und ein nationaler Ärzteverband: 4 Jahre VSAO Sektion Bern, 9 Jahre Ärztegesellschaft des Kantons Bern, im 4. Jahr Verbindung der Schweizer Ärzte

Praxisführung: 24 Jahre Grundversorger-Zweierpraxis Fraktionsführung: 4 Jahre im Gemeindeparlament von Zollikofen

\section{Motivation}

Wo Menschen gemeinsam handeln, entsteht politische Stärke. Mit meiner Kandidatur für den Zentralvorstand und dessen Vorsitz will ich weiterhin dazu beitragen, die Kräfte innerhalb der Ärzteschaft zu bündeln. Wenn der Zusammenhalt zwischen Ärztinnen und Ärzten der verschiedenen Sprachregionen und unterschiedlichen Fachdisziplinen gross ist, nimmt die Politik unsere Stimme ernst. - Ich kämpfe für die Freiheiten des Arztberufes, denn die Verantwortung, die wir übernehmen, erfordert die dazu notwendige Freiheit. Ich will eine Reduktion der administrativen Belastung, denn diese demotiviert. Ich stehe ein für attraktive Rahmenbedingungen für Ärztinnen und Ärzte in Spital und Praxis; dazu gehören Arbeitsmodelle, die heutigen Lebensentwürfen entsprechen. Und ich lehne umfassende Leistungsversprechen der Krankenversicherer $a b$, wenn diese Versprechen durch gleichzeitige Sparvorgaben unerfüllbar werden. - Erfolgreiche Zusammenarbeit erfordert für mich gegenseitiges Vertrauen und Respekt. Ich freue mich auf die Zukunft und auf sachgerechte, umsetzbare Lösungen. 


\section{Urs Stoffel (bisher)}

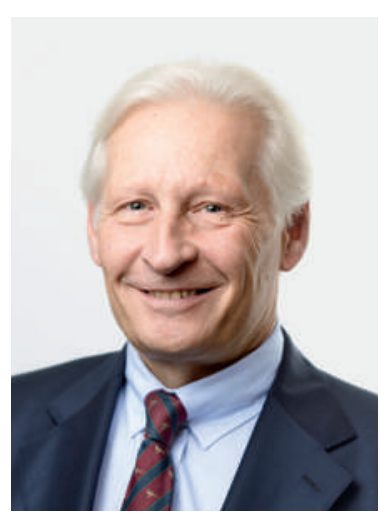

Titel: Dr. med.

Geburtsjahr: 1952

Wohnort: Kilchberg ZH

Bürgerort: Arbon TG

Zivilstand: verheiratet, zwei Töchter

E-Mail: urs.stoffel[at]hin.ch

Facharzttitel: Allgemeine Chirurgie

\section{Mitglied bei medizinischen Gesellschaften}

Schweizerische Gesellschaft für Chirurgie (SGC), Schweizerische Arbeitsgemeinschaft für laparoskopische und thorakoskopische Chirurgie (SALTC), Schweizerische Gesellschaft für Allgemeinchirurgie und Traumatologie (SGACT), Deutsche Gesellschaft für Koloproktologie DGK e.V., Verband der chirurgisch und invasiv tätigen Fachgesellschaften fmCh, Gesellschaft der Chirurgen des Kantons Zürich (Mitglied und Quästor)

\section{Interessenbindungen}

Verwaltungsratspräsident der NewIndex AG (im Rahmen des Mandats im FMH-Zentralvorstand), Verwaltungsratspräsident der HIN AG (im Rahmen des Mandats im FMH-Zentralvorstand), Stiftungsrat der «Fondation Gaydoul», Stiftungsrat der Stiftung der ehemaligen Höhenklinik Sanitas Davos, Verwaltungsrat der RehaClinic Zürich AG, Vorstandsmitglied des Vereins "Zurich Affinity Domain» (im Mandat der Ärztegesellschaft des Kantons Zürich AGZ)

\section{Weitere Qualifikationen}

Handelsdiplom

\section{Sprachen}

Deutsch: Muttersprache; Französisch: Kenntnisse; Italienisch: Kenntnisse, Englisch: gute Kenntnisse

\section{Aktuelle berufliche Tätigkeit}

Niedergelassener Allgemeinchirurg in der chirurgischen Gemeinschaftspraxis Enge in Zürich mit Belegarzttätigkeit an vier Kliniken (Klinik Hirslanden, Klinik Im Park, Privatklinik Bethanien und Seespital Kilchberg)

\section{Parteizugehörigkeit}

FDP

\author{
Bisherige politische Mandate \\ Keine
}

\section{Bisherige standespolitische Mandate}

Mitglied des Zentralvorstands der FMH, seit 2012 (Departement eHealth Sicherheitsinfrastruktur und Datenerhebung, seit März 2015 Departement ambulante Tarife und Verträge Schweiz a.i.), Präsident der Ärztegesellschaft des Kantons Zürich AGZ, von 2002-2014, Co-Präsident der Konferenz der Kantonalen Ärztegesellschaften KKA-CCM, von 2006-2011, Delegierter der FMH im Kostenneutralitätsbüro TARMED, 2004

\section{Führungserfahrung}

S. «Bisherige standespolitische Mandate»

\section{Motivation}

In den letzten vier Jahren habe ich zuerst das Departement eHealth - Sicherheitsinfrastruktur und Datenerhebung der FMH betreut. Ein wichtiger Meilenstein war der Wechsel von der Swisscom zur ärzteeigenen Firma HIN AG als Provider für die Health Professional Card (HPC). In naher Zukunft werden wir für das elektronische Patientendossier (EPD) die Identifizierung und Authentifizierung der Ärztinnen und Ärzte durch die HPC zusätzlich in Richtung einer elektronischen Identifikation (eID) weiterentwickeln. Seit März 2015 habe ich nach dem überraschenden Tod des FMH-Vizepräsidenten Ernst Gähler zusätzlich das Departement Ambulante Tarife und Verträge Schweiz übernommen. Hier laufen die Arbeiten zum Abschluss der Revision der ambulanten Tarifstruktur auf Hochtouren. Es ist mir ein grosses Anliegen, die Tarifautonomie für die schweizerische Ärzteschaft zu erhalten und einen Staatstarif zu verhindern. Gerne stelle ich mich für eine weitere Legislatur als Mitglied des Zentralvorstands der FMH zur Verfügung. 


\section{Jean-Claude Brückner (bisher)}

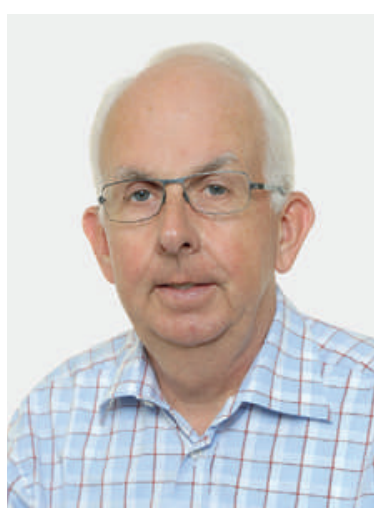

Titel: Dr.

Geburtsjahr: 1954

Wohnort: Genf

Heimatort: Genf

Zivilstand: verheiratet

E-Mail: jcbruckner[at]bluewin.ch

Facharzttitel: Anästhesiologie und Reanimation

Mitgliedschaft bei medizinischen Gesellschaften Ärztegesellschaft des Kantons Genf (AMG)

Sprachen

Französisch
Bisherige standespolitische Mandate

Ehemaliger Präsident der Genfer Gesellschaft der Anästhesisten (SMAG), ehemaliger Präsident des Verwaltungsrats des Trust Centers von CTESIAS, ehemaliges Mitglied des Verwaltungsrats von NewIndex, Mitglied des Rats der AMG von 2003 bis 2015, Mitglied des Büros der AMG von 2007 bis 2013 (als Kassier), Mitglied der Begleitkommission für Wirtschaftlichkeitsverfahren der AMG seit 2010

\section{Motivation}

Nach aktiver Mitgliedschaft in verschiedenen Vereinsvorständen beteilige ich mich nun mit grossem Interesse an der Arbeit der Geschäftsprüfungskommission der FMH und möchte dieses Engagement fortsetzen. 


\section{Jürg Lareida (bisher)}

Geburtsjahr: 1958

Wohnort: Aarau

Bürgerort: Aarau und Präz

Zivilstand: verheiratet

E-Mail: juerg.lareida[at]hin.ch

Facharzttitel: Endokrinologie und Diabetologie,

Allgemeine Innere Medizin

\section{Mitglied bei medizinischen Gesellschaften}

Schweizerische Gesellschaft für Endokrinologie und Diabetologie (SGED); Schweizerische Gesellschaft für Allgemeine Innere Medizin (SGAIM); Schweizerische Vereinigung gegen die Osteoporose (SVGO); European Society of Endocrinology; Endocrine Society; European Group of Graves' Orbitopathy

Interessenbindungen

Keine

\section{Sprachen}

Deutsch, Französisch, Englisch, Italienisch

Aktuelle berufliche Tätigkeit

Privatärztliche Tätigkeit, Belegarzt

\section{Parteizugehörigkeit}

Keine

\section{Bisherige politische Mandate}

Einwohnerrat der Stadt Aarau 1991-1997, damals Mitglied der Finanz- und Geschäftsprüfungskommission der Stadt Aarau; Steuerkommission der Stadt Aarau 1999-2004

\section{Bisherige standespolitische Mandate}

Mitglied der Geschäftsleitung des Aargauischen Ärzteverbandes; Mitglied der Geschäftsprüfungskommission der FMH seit 2013

\section{Motivation}

Ich durfte bereits in den letzten dre Jahren Mitglied in der Geschäftsprüfungskommission sein. Gerne stelle ich mich für eine weitere Amtsperiode zur Verfügung. 


\section{Thomas Kehl (bisher)}

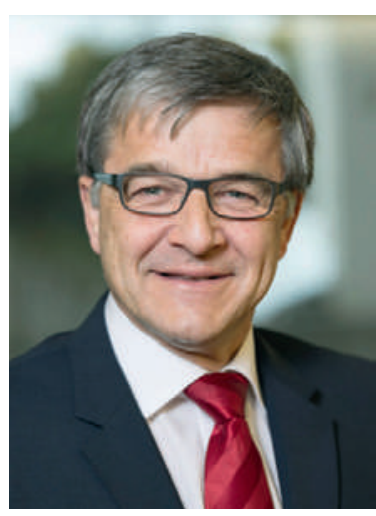

Titel: Dr. med.

Geburtsjahr: 1953

Wohnort: 7272 Davos Clavadel

Bürgerort: Rebstein SG

Zivilstand: verheiratet

E-Mail: thomas.kehl[at]zhreha.ch

Facharzttitel: Orthopädische Chirurgie und

Traumatologie

Mitglied bei medizinischen Gesellschaften

swiss orthopaedics

Interessenbindungen

Keine

Weitere Qualifikationen

MBA

Sprachen

Deutsch, Französisch, Englisch, Schwedisch

\section{Aktuelle berufliche Tätigkeit}

Vorsitzender der Direktion Zürcher RehaZentren

Parteizugehörigkeit

FDP

Bisherige politische Mandate

Keine

Bisherige standespolitische Mandate

Mitglied der Geschäftsprüfungskommission der FMH; seit 18 Jahren Vorsitzender der Direktion Zürcher RehaZentren mit 600 Mitarbeitenden

\section{Führungserfahrung}

Jahrelange Tätigkeit als Orthopädischer Chirurge in leitenden Positionen (u.a. auch Chefarzt); CEO der Zürcher RehaZentren

\section{Motivation}

Motivation für meine erneute Kandidatur als Mitglied der Geschäftsprüfungskommission der FMH ist die weitere Mitarbeit an einer standespolitisch überzeugenden, leistungsfähigen Ärztegemeinschaft auf einer betriebswirtschaftlich gesunden Basis. 


\section{Adrian Sury (bisher)}

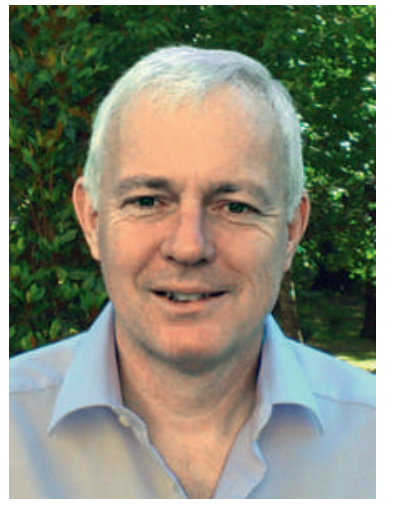

Titel: Dr. med.

Geburtsjahr: 1955

Wohnort: 6602 Muralto TI

Bürgerort: Locarno TI, Wiler b. U. BE und Italien

Zivilstand: verheiratet, drei Kinder

E-Mail: Adrian.Sury[at]hin.ch,

a.sury[at]clinicasantachiara.ch

Facharzttitel: Allgemeine Innere Medizin

Mitglied bei medizinischen Gesellschaften

Schweizerische Gesellschaft für Allgemeine Innere Medizin SGAIM, Schweizerische Gesellschaft für Arbeitsmedizin SGARM, Schweizerische Gesellschaft für Musik-Medizin SMM, Ordine dei Medici del Cantone Ticino OMCT, Circolo medico del Verbano, Pardomed

\section{Interessenbindungen}

Keine

\section{Weitere Qualifikationen}

Betriebsarzt SGARM

Sprachen

Italienisch, Deutsch, Französisch, Englisch

\section{Aktuelle berufliche Tätigkeit}

Hausarztpraxis Al Lido, Belegarzttätigkeit und ärztlicher Direktor der Clinica Santa Chiara, Locarno, Weiterbildungsverantwortlicher für Allgemeine Innere Medizin Clinica Santa Chiara

\section{Parteizugehörigkeit}

CVP

\section{Bisherige politische Mandate}

Keine

\section{Bisherige standespolitische Mandate}

Ehemaliger Kassier und gewesenes Vorstandsmitglied des Ordine dei Medici del Cantone Ticino OMCT, Mitglied der ehemaligen Finanzkommission bzw. der heutigen Geschäftsprüfungskommission der FMH

\section{Führungserfahrung}

Ärztlicher Direktor und Verwaltungsratsvizepräsident Clinica Santa Chiara, Verantwortlicher für Weiterbildung in Allgemeiner Innerer Medizin Clinica Santa Chiara (Kategorie B, 2 Jahre), Consiglio di gestione OMCT, Präsident Accademia Vivaldi della Svizzera Italiana (kantonal anerkannte Musikschule)

\section{Motivation}

Seit 1997 zuerst in der Finanzkommission der FMH, nach der Strukturreform in deren Nachfolgegremium, der FMH-Geschäftsprüfungskommission, engagiert, in den letzten vier Jahren als Präsident, möchte ich nochmals für eine Legislaturperiode kandidieren. Verschiedene Baustellen sind zurzeit offen und aktuell, wie etwa die Umsetzung der von der Ärztekammer 2015 beschlossenen Budgetstabilisierungsmassnahmen, die zukünftige Publikationsstrategie der $\mathrm{FMH}$, die Rolle der Beteiligungsgesellschaften und andere mehr. Um diese Aufgaben konkret und lösungsorientiert anzugehen, ist das Wissen um deren historischen Hintergrund von grossem Wert. Als Allgemeininternist, der in der eigenen Hausarztpraxis, als Belegarzt und als ärztlicher Direktor und Fortbildungsverantwortlicher tätig ist, denke ich, die notwendige Unabhängigkeit und den Weitblick einer 35-jährigen Berufserfahrung weiterhin in der Geschäftsprüfungskommission der FMH einbringen zu können. 


\section{Alexander Zimmer (bisher)}

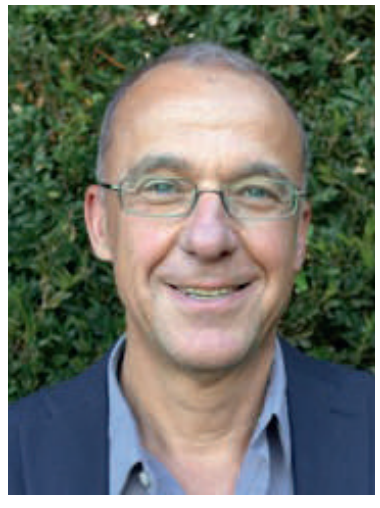

Titel: Dr. med.

Geburtsjahr: 1961

Wohnort: Solothurn

Bürgerort: Zürich/ZH

Zivilstand: in Partnerschaft lebend, zwei Töchter E-Mail: azimmer[at]hin.ch

Facharzttitel: Psychiatrie und Psychotherapie,

Schwerpunkt Konsiliar- und Liaisonpsychiatrie

\section{Mitglied bei medizinischen Gesellschaften}

FMPP (Foederatio Medicorum Psychiatricorum et Psychotherapeuticorum); SGPP (Schweizerische Gesellschaft für Psychiatrie und Psychotherapie); SSCLP (Swiss Society of Consultation - Liaison Psychiatry); SSAM (Swiss Society of Addiction Medicine); GAeSO (Gesellschaft der Ärztinnen und Ärzte des Kantons Solothurn); GPPSO (Gesellschaft für Psychiatrie und Psychotherapie des Kantons Solothurn)

\section{Interessensverbindungen}

Keine

\section{Weitere Qualifikatione}

2008-2009: Neue Konzepte des VerwaltungsratsManagements, Institut für Führung und Personalmanagement, Prof. Dr. Martin Hilb, Universität St. Gallen; 2008: ERFA-Tagungen für Verwaltungsräte und Leitungen von Spitälern: Führung und Aufsicht von Spitälern; Universität St. Gallen; 1999-2000: Managementweiterbildung am College M, Bern;

\section{Sprachen}

Deutsch Muttersprache, Französisch und Englisch gut in Wort und Schrift

\section{Aktuelle berufliche Tätigkeit}

Seit 2014 in eigener Praxis in Solothurn

\section{Bisherige standespolitische Mandate}

Seit 2012 Mitglied der Geschäftsprüfungskommission (GPK) der FMH; seit 2013 Ersatzmitglied der FMH-Ärztekammer (für die FMPP) und seit 2014 der FMH AG DDQ (Daten Demographie und Qualität) (für die FMPP); seit 2014 Mitglied der FMH AG eHealth (für die FMPP); seit 2003 Mitglied der Ständigen Tarifkommission (STK) der FMPP, seit 2013 deren Stv. Präsident; 2009-2014: Vorstandsmitglied der Schweizerischen Vereinigung Psychiatrischer Chefärzte und Chefärztinnen (SVPC); 2006-2014: Vorstandsmitglied der Fachgruppe Psychiatrie Baselland; 2006-2014: Delegierter der Fachgruppe Psychiatrie Baselland / der SVPC an den Delegiertenversammlungen von FMPP und SGPP

\section{Führungserfahrung}

1997 bis 2014 in leitenden Funktionen im Spitalbereich: Oberarzt und Leitender Arzt im Kanton Solothurn; Chefarzt und Geschäftsleitungsmitglied der Psychiatrie Baselland

\section{Motivation}

Die Arbeit in der Geschäftsprüfungskommission der FMH (GPK) ist anspruchsvoll. Es braucht einige Zeit, bis man sich in die Geschäfte der Delegiertenversammlung, des Zentralvorstandes, des Schweizerischen Institutes für ärztliche Weiter- und Fortbildung (SIWF) sowie des Generalsekretariats eingearbeitet hat, die beteiligten Menschen kennen gelernt hat und der Ärztekammer gegenüber entsprechend Rechenschaft ablegen kann. Die Mitglieder der GPK sind während der vergangenen Amtsperiode zu einem effizient und konstruktiv arbeitenden Team zusammengewachsen. Gerne würde ich mich deshalb für eine weitere Amtsdauer von vier Jahren als Geschäftsprüfungskommissionsmitglied zur Verfügung stellen. 


\section{Francesca Mainieri}

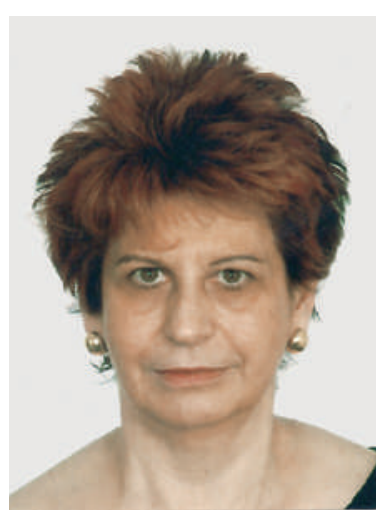

Titel: Dr. med.

Geburtsjahr: 1957

Wohnort: 6933 Muzzano

Bürgerort: Zürich

Zivilstand: ledig

E-Mail: francesca.mainieri[at]hin.ch

Facharzttitel: Allgemeine Innere Medizin

Mitglied bei medizinischen Gesellschaften

Schweizerische Gesellschaft für Allgemeine Innere Medizin (SGAIM); Associazione Medici Internisti Ticinesi (AMITI)

Interessenbindungen

Keine

Sprachen

Italienisch, Deutsch, Französisch

Aktuelle berufliche Tätigkeit

Eigene Arztpraxis

\section{Parteizugehörigkeit \\ Keine}

\section{Bisherige politische Mandate}

Keine, aber intensive langjährige Zusammenarbeit mit verschiedenen Politikern und Institutionen im Kanton Tessin

\section{Bisherige standespolitische Mandate}

Präsidentin Associazione Medici Internisti Ticinesi (AMITI)

\section{Führungserfahrung}

Langjährige selbstständige Praxistätigkeit; 25 Jahre lang im Vorstand der AMITI, seit 1990 Präsidentin

\section{Motivation}

In meinem Berufsleben habe ich mich immer zur Lösung von sozialen und medizinischen Problemen verpflichtet gefühlt und habe dabei viel Erfahrung gesammelt, die ich gerne auch in Zukunft weitergeben möchte. Als Ärztin und als Vertreterin der italienischen Schweiz glaube ich, dass ich noch vieles für unsere Gesellschaft tun kann und würde mich sehr freuen, wenn ich wieder eine Gelegenheit dazu bekäme. 


\section{Jean-Baptiste Ollyo}

Titel: Dr. med.

Geburtsjahr: 1950

Wohnort: Préverenges (VD)

Zivilstand: verheiratet

E-Mail: jbollyo[at]hotmail.com

Facharzttitel: Allgemeine Innere Medizin

und Gastroenterologie

\section{Mitglied bei medizinischen Gesellschaften}

Ärztegesellschaft des Kantons Waadt, Schweizerische Gesellschaft für Gastroenterologie, Schweizerische Gesellschaft für Ultraschall in der Medizin, Fraternité AghPan (humanistische Medizin unter Wahrung der staatsbürgerlichen Verantwortung)

\section{Weitere Qualifikationen}

Medizinrecht

\section{Sprachen}

Französisch, Italienisch, Englisch, gute Deutschkenntnisse

\section{Aktuelle berufliche Tätigkeit}

Arzt in Teilzeit in einem medizinischen Zentrum, Verfassen eines Buches über konstitutionelle Magerkeit

\section{Motivation}

Ich kandidiere für das Amt des Präsidenten und ersatzweise für das Amt des Vizepräsidenten der Standeskommission der FMH, um:

1. mich für eine echte Professionalisierung der Standeskommission der FMH einzusetzen;

2. die Befugnisse der Standeskommission auszubauen;

3. eine strikte Gewaltentrennung einzuführen und eine Ämterhäufung zu verbieten;

4. die Information zu verbessern (alle standesrechtlichen Entscheide, wie die BGE, ausnahmslos für jeden zugänglich zu machen);

5. die ethischen und berufsständischen Werte zu verteidigen und nicht bloss das Standesimage ...;

6. mich dafür einzusetzen, dass alle Mitglieder des Zentralvorstands der FMH wirklich integer, unabhängig und unparteiisch sind, ohne Mauscheleien, und sich an die übergeordneten Texte halten, auch an die administrativen;

7. Personen, die nicht Mitglied der FMH oder einer kantonalen Ärztegesellschaft sind, die Möglichkeit einzuräumen, sich bei Bedarf an die Standeskommission der FMH zu wenden;

8. einen ehemaligen Zivilrichter als Autorität in die Standeskommission der FMH aufzunehmen.

\section{Parteizugehörigkeit}

FDP. Die Liberalen 